8th Alexander Friedmann International Seminar

on Gravitation and Cosmology

International Journal of Modern Physics: Conference Series

Vol. 3 (2011) 294-302

(C) World Scientific Publishing Company

DOI: $10.1142 /$ S2010194511001395

\title{
BOUNCING MODELS WITH A COSMOLOGICAL CONSTANT
}

\author{
NELSON PINTO-NETO, BEATRIZ B. SIFFERT, RODRIGO MAIER \\ and STELLA PEREIRA \\ ICRA - Centro Brasileiro de Pesquisas Físicas - CBPF \\ Rua Dr. Xavier Sigaud, 150, Urca, CEP 22290-180, Rio de Janeiro, Brazil \\ nelsonpn@cbpf.br, beatriz@cbpf.br,rodmaier@cbpf.br, stella@cbpf.br
}

Received 3 June 2011

\begin{abstract}
Most bouncing models contain a contracting phase from a very large and rarefied state, where dark energy might have had an important role. If this is that case, the presence of dark energy can modify the initial conditions and evolution of cosmological perturbations, changing the known results already obtained in the literature concerning their amplitude and spectrum. In this work, we assume the simplest and most appealing candidate for dark energy, the cosmological constant, and study its influence on the evolution of cosmological perturbations during the contracting phase of a bouncing model, containing also a perfect fluid with constant equation of state parameter $w$. We show that, due to the vacuum state choice we have to make when a cosmological constant is present, the spectrum of the perturbations are substantially altered. We conclude that, in this case, the presence of a stiff matter fluid in the contracting phase is needed in order to have a scale invariant spectrum of perturbations in the expanding phase.
\end{abstract}

Keywords: Bouncing models; cosmological constant; cosmological perturbations.

PACS numbers: 98.80.Cq, 04.60.Ds

\section{Introduction}

The present phase of accelerated expansion of the Universe can be explained by the existence of a well suited cosmological constant. The so-called $\Lambda$ CDM standard model assumes that there exists a cosmological constant term in Einstein's equations, which becomes dynamically important when the typical scale of the Universe has the size of the present Hubble radius.

Bouncing cosmological models, ${ }^{1-6}$ which solve the singularity problem, can also be considered as possible alternatives to inflation, as long as they are able to solve the horizon and flatness problems, and justify the observed power spectrum of primordial cosmological perturbations.

If the contracting phase of a regular bouncing model is dominated by a perfect fluid, it has been shown by many authors, in different frameworks, ${ }^{7-12}$ that this fluid must be dust, in order to reproduce a scale invariant spectrum of scalar and tensor cosmological perturbations. 
In the case with no cosmological constant, we can set the vacuum initial conditions for quantum cosmological perturbations in the far past of the contracting phase, when the Universe was very big and almost flat, justifying the choice of an adiabatic Minkowski vacuum in that phase. However, if a cosmological constant is present, the asymptotic past of bouncing models will approach De Sitter rather than Minkowski spacetime. The existence of a cosmological constant can, therefore, modify the spectrum and amplitude of cosmological perturbations substantially.

In the present work we study in detail the issue of bouncing models in the context of a Friedmann-Lemaître-Robertson-Walker geometry with a cosmological constant and a perfect fluid satisfying a constant equation of state $p=w \epsilon$. The bounce itself takes place due to quantum effects when the Universe was very small and entirely dominated by the fluid. Hence, the presence of the cosmological constant does not modify the evolution of the background and perturbations in that period, and the description provided in Refs. 11, 13-15 can still be considered to be valid at the bounce. This should also be valid for other bouncing models, ${ }^{7-11}$ as long as any bounce should take place at very short length scales, where the cosmological constant has no role.

In the next section, we will settle down the background model and obtain the equations for the evolution of cosmological perturbations on this background. In Sec. 3 we will make a discussion about the choice of initial state of the cosmological perturbations on this background. In Sec. 4 we will obtain analytically the power spectrum of perturbations and discuss its physical consequences. We end up with discussions and conclusions.

\section{Background Model and Equations for Scalar Perturbations}

We begin with the gravitational action of General Relativity with a cosmological constant,

$$
\mathcal{S}_{\mathrm{GR}}=-\frac{1}{6 \ell_{\mathrm{Pl}}^{2}} \int \sqrt{-g}(R+2 \Lambda) \mathrm{d}^{4} x,
$$

where $\ell_{\mathrm{P} 1}=\left(8 \pi G_{\mathrm{N}} / 3\right)^{1 / 2}$ is the Planck length in natural units $(\hbar=c=1)$, and $\Lambda$ is the cosmological constant, and the spatially flat homogeneous and isotropic line element,

$$
\mathrm{d} s^{2}=a^{2}(\eta)\left(\mathrm{d} \eta^{2}-\delta_{i j} \mathrm{~d} x^{i} \mathrm{~d} x^{j}\right) .
$$

The matter content of the model is described by the action (see Ref. 16 for details)

$$
S_{m}=\int p(\chi, \varphi) d^{4} x,
$$

where $\varphi$ is a scalar field with kinetic term

$$
\chi=\frac{1}{2} g^{\alpha \beta} \varphi, \alpha \varphi, \beta,
$$


and $p$ plays the role of a pressure. By varying (3) with respect to the metric, we obtain the energy-momentum tensor

$$
T_{\nu}^{\mu}=(\epsilon+p) V^{\mu} V_{\nu}-p \delta_{\nu}^{\mu},
$$

with $V_{\nu} \equiv \varphi,{ }_{\nu} / \sqrt{2 \chi}$, and $\epsilon \equiv 2 \chi p,{ }_{\chi}-p\left(p,,_{\chi} \equiv \partial p / \partial \chi\right)$, which plays the role of an energy density.

For the case of a perfect fluid with $p=w \epsilon$ and $w$ constant, the solution for the scale factor in terms of cosmic time $d t=a d \eta$ reads,

$$
a(t)=a_{0}\left(\frac{\Omega_{0 \omega}}{\Omega_{\Lambda}}\right)^{1 / 3(1+\omega)}\left[\sinh \left(-\frac{3 \sqrt{\Omega_{\Lambda}}(1+\omega) H_{0}}{2} t\right)\right]^{2 / 3(1+\omega)},
$$

where $H_{0}=72 \mathrm{~km} \mathrm{~s}^{-1} \mathrm{Mpc}^{-1}$ is the present Hubble parameter, $\Omega_{0 \omega} \equiv \epsilon_{0} / \epsilon_{\text {crit }}$ with $\epsilon_{\text {crit }} \equiv 3 H_{0}^{2} /(8 \pi G)$, and $\Omega_{\Lambda} \equiv \Lambda / H_{0}^{2}$.

The subscript 0 indicates the present values of the respective quantities.

Due to the constraint equations present in the Einstein's equations, the evolution of quantum perturbations in a classical background is described by a single quantum field, the gauge invariant Mukhanov-Sasaki variable, defined by

$$
v \equiv \sqrt{\epsilon, \chi} a\left(\delta \varphi+\frac{\varphi_{\text {back }}^{\prime}}{\mathcal{H}} \psi\right)
$$

where $\delta \varphi$ is the perturbed scalar field, and $\varphi_{\text {back }}$ is its background solution.

The Mukhanov-Sasaki variable satisfies the equation,

$$
v^{\prime \prime}-c_{s}^{2} \nabla^{2} v-\frac{z^{\prime \prime}}{z} v=0
$$

where

$$
z \equiv \frac{a^{2} \sqrt{\epsilon+p}}{c_{s} \mathcal{H}}
$$

and

$$
c_{s}^{2} \equiv \frac{p, \chi}{\epsilon, \chi} .
$$

For a perfect fluid with $p=w \epsilon$ and the scale factor given by Eq. (6), we obtain

$$
\begin{gathered}
c_{s}^{2}=w, \\
z(t)=-\sqrt{\frac{3}{2}\left(\frac{1+w}{w}\right)} \frac{a(t)}{\cosh (\gamma t)},
\end{gathered}
$$

and

$$
V(t) \equiv \frac{z^{\prime \prime}}{z}=\frac{\Omega_{\Lambda}}{R_{H}^{2}(1+z)^{2}}\left\{\frac{(1-3 w)}{2}\left[\frac{1}{\sinh ^{2}(\gamma t)}-\frac{(1+3 \omega)}{2}\right]-\frac{9(1+w)^{2}}{2 \cosh ^{2}(\gamma t)}\right\},
$$

where

$$
\gamma \equiv \frac{3 \sqrt{\Omega_{\Lambda}}(1+\omega) H_{0}}{2}
$$




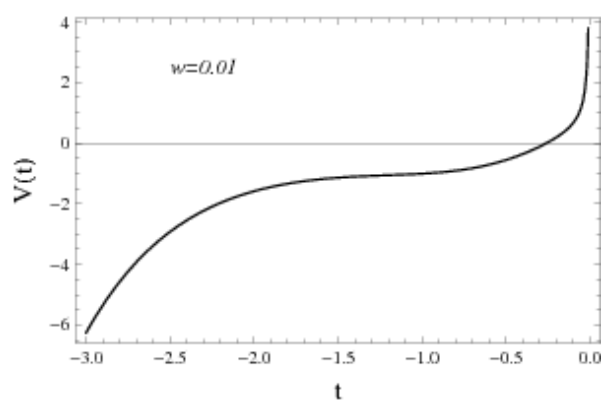

Fig. 1. Behaviour of the potential $V(t)$ given by Eq. (13) for $w=0.01$. Here we used $a_{0}=H_{0}=1$.

$R_{H} \equiv 1 /\left(a_{0} H_{0}\right)$ is the co-moving Hubble radius, $1+z=a_{0} / a, z$ being the red-shift (not the $z$ defined in Eq. (12)), and $a$ is given by Eq. (6).

For $t \rightarrow-\infty$, Eq. (6) yields $a(t) \propto \exp (-\sqrt{\Lambda} t)$ or, in conformal time, $a(\eta) \approx$ $1 /\left[\sqrt{\Lambda}\left(\eta+\eta_{\infty}\right)\right]$, where $-\eta_{\infty}<\eta \ll 0$, and $\eta_{\infty}$ is a positive constant. This corresponds to the usual De Sitter behaviour. In this case, potential (13) reads

$$
V(\eta) \equiv \frac{z^{\prime \prime}}{z} \approx \frac{9 w^{2}-1}{4\left(\eta+\eta_{\infty}\right)^{2}} .
$$

For $t \rightarrow 0^{-}$, or $\eta \rightarrow 0^{-}$, we have $a(t) \propto t^{2 /[3(1+w)]}$ or, in conformal time, $a(\eta) \propto \eta^{2 /(1+3 w)}$, which corresponds to the usual perfect fluid Friedmann evolution. In this regime, we obtain

$$
V(\eta) \equiv \frac{z^{\prime \prime}}{z} \approx \frac{2(1-3 w)}{(1+3 w)^{2} \eta^{2}} .
$$

In Figs. 1-3 we present the behaviour of the potential $V(t)$ for $t<0$ in the cases $w<1 / 3, w=1 / 3$ and $w>1 / 3$.

We will now check if the singularity at $t=0$, which separates the contracting and expanding solutions, can be eliminated if we evoke the quantum cosmological

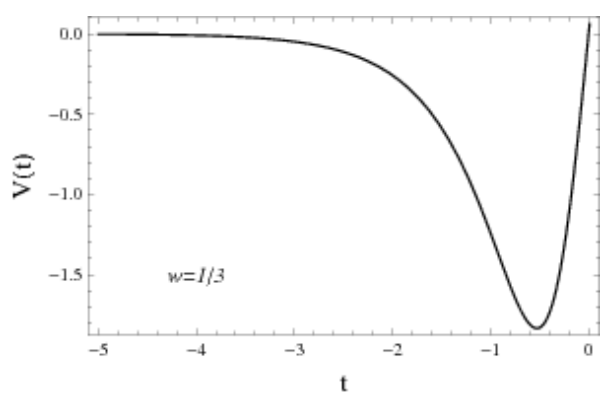

Fig. 2. The same as Fig. 1 for $w=1 / 3$. 


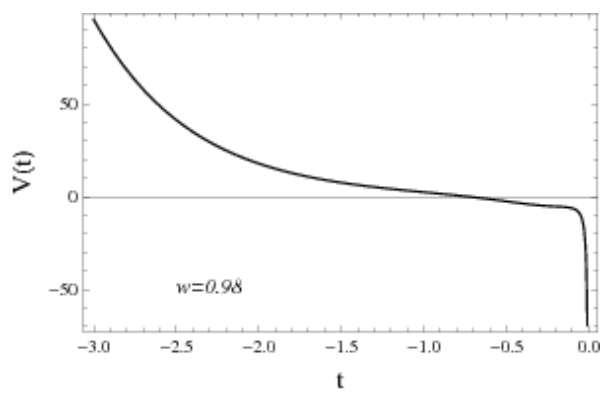

Fig. 3. The same as Fig. 1 for $w=0.98$.

bounces with a perfect fluid studied in Refs. 11, 13-15. These solutions present a regular scale factor given by

$$
a(T)=a_{b}\left[1+\left(\frac{T}{T_{b}}\right)^{2}\right]^{\frac{1}{3(1-\omega)}},
$$

where $d T=a^{1-3 w} d \eta$, and $a_{b}$ and $T_{b}$ are positive constants. Note that for $|T| \gg T_{b}$, this solution approaches the classical Friedmann solution for a perfect fluid. Hence, the scale factor (6) can be smoothly connected to the scale factor (17).

\section{Choice of Initial State for Quantum Perturbations}

It was shown in Ref. 15 that perturbations around these quantum bouncing solutions satisfy

$$
v^{\prime \prime}-c_{s}^{2} \nabla^{2} v-\frac{a^{\prime \prime}}{a} v=0,
$$

which tends to the potential $V(t)$ presented in Eq. (16) in the limit $|T| \gg T_{b}$, but does not diverge in $\eta=0$. The quantum bouncing potential $a^{\prime \prime} / a$, obtained from Eq. (17) is presented in Figs. $4-6$ for the cases $w<1 / 3, w=1 / 3$ and $w>1 / 3$.

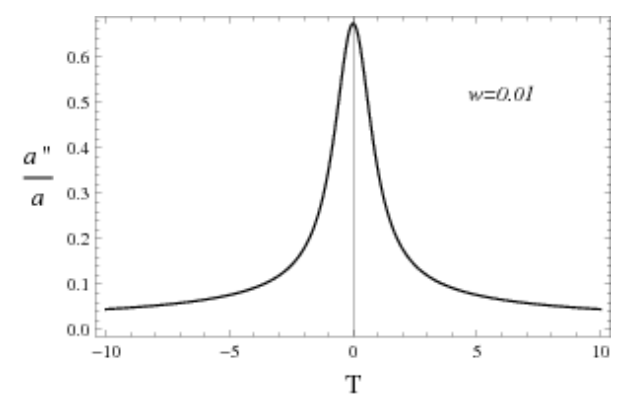

Fig. 4. Behaviour of the quantum bouncing potential given by Eq. (17) for $w=0.01$ Here we used $a_{b}=T_{b}=1$. 


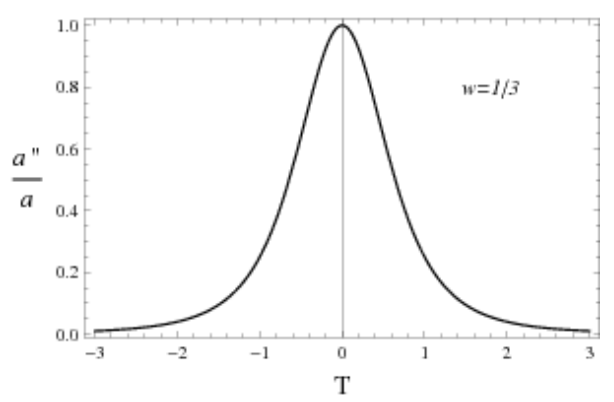

Fig. 5. The same as Fig. 4 for $w=1 / 3$.

When these quantum effects become important near $\eta=0$ producing the bounce, the two disjoint parts of the classical potentials presented in Figs. 1-3 can be softly connected with the potentials presented in Figs. 4-6. It is possible, therefore, to smoothly evolve the perturbations from the contracting phase to the expanding phase, and calculate their properties in the present era.

In order to realize this program, one must set the initial conditions for the perturbations in the past contracting phase. Without the cosmological constant, the Universe tends, in the far past, to Minkowski spacetime, where the potentials become null (see Fig. 2). In the presence of the cosmological constant, neither does the Universe tend to Minkowski spacetime in the far past (in fact, it tends to De Sitter spacetime), nor does the potential become null there (except for $w=1 / 3$ ).

Although we cannot use a Minkowski vacuum to set our initial conditions, there is a time region in the contracting phase of the model where a special quantum state can be defined. Note that in the asymptotic past, the Universe approaches De Sitter spacetime. At this stage, the equation for the Mukhanov-Sasaki variable reads (see Eq. (15))

$$
v_{k}^{\prime \prime}+\left[w k^{2}-\frac{\left(9 w^{2}-1\right)}{4\left(\eta+\eta_{\infty}\right)^{2}}\right] v_{k}=0 .
$$

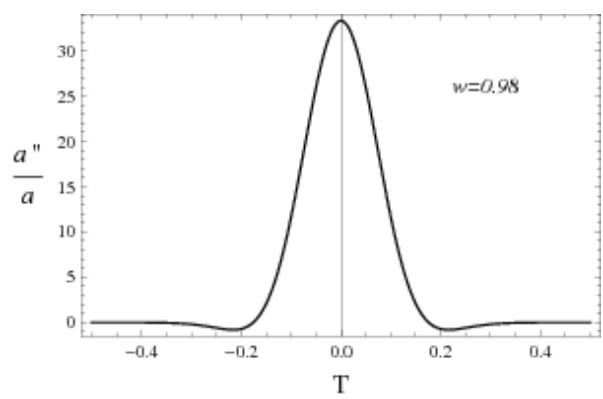

Fig. 6. The same as Fig. 4 for $w=0.98$. 
This equation is completely equivalent to an equation for a massive scalar field in a De Sitter spacetime, with mass given by

$$
m=\frac{3 \sqrt{\Lambda}}{2} \sqrt{1-w^{2}}
$$

Its general solution reads

$$
v_{k}=\sqrt{\eta}\left[c_{1} H_{\nu}^{(1)}(\sqrt{w} k \eta)+c_{2} H_{\nu}^{(2)}(\sqrt{w} k \eta)\right],
$$

where the $H_{\nu}^{(1,2)}$ are the Hankel functions of first and second kind, $\nu=3 w / 2$, and we have made a conformal time translation $\left(\eta+\eta_{\infty}\right) \rightarrow \eta$.

The mode function with $c_{1}=1$ and $c_{2}=0$ determines the so=called BunchDavies vacuum. This special state is a time independent state, in the sense that the amplitude of fluctuations on a given physical scale does not depend on time (see Ref. 17 for details), and it coincides with the Minkowski vacuum for $\sqrt{w} k \eta \gg 1$.

We can now calculate the spectrum of perturbations for this choice of initial state.

\section{Spectrum of Quantum Cosmological Perturbations}

In the region where Eq. (19) is valid $\left(\left|\eta+\eta_{\infty}\right| \ll 1\right)$, for the interesting interval $1<k<10^{3}$, the argument of the Bunch-Davies solution

$$
v_{k}=\sqrt{\eta} H_{\nu}^{(1)}(\sqrt{w} k \eta)
$$

is very small (remember we made the time translation $\left(\eta+\eta_{\infty}\right) \rightarrow \eta$ ). Its series expansion reads

$$
\lim _{\eta \rightarrow 0} v_{k}=a_{1} \eta^{(1+3 w) / 2} k^{3 w / 2}+a_{2} \eta^{(1-3 w) / 2} k^{-3 w / 2},
$$

where $a_{1}$ and $a_{2}$ are constants which do not depend on $k$.

On the other hand, the complete solution of equation

$$
v_{k}^{\prime \prime}+\left(w k^{2}-\frac{z^{\prime \prime}}{z}\right) v_{k}=0
$$

can also be expanded in powers of $k^{2}$ according to the formal solution ${ }^{18}$

$$
\begin{aligned}
\frac{v}{z} & \simeq A_{1}(k)\left[1-\omega k^{2} \int^{\eta} \frac{\mathrm{d} \bar{\eta}}{z^{2}(\bar{\eta})} \int^{\bar{\eta}} z^{2}(\overline{\bar{\eta}}) \mathrm{d} \overline{\bar{\eta}}\right] \\
& +A_{2}(k)\left[\int^{\eta} \frac{\mathrm{d} \bar{\eta}}{z^{2}(\bar{\eta})}-\omega k^{2} \int^{\eta} \frac{\mathrm{d} \bar{\eta}}{z^{2}(\bar{\eta})} \int^{\bar{\eta}} z^{2}(\overline{\bar{\eta}}) \mathrm{d} \overline{\bar{\eta}} \int^{\overline{\bar{\eta}}} \frac{\mathrm{d} \overline{\bar{\eta}}}{z^{2}(\overline{\bar{\eta}})}\right],
\end{aligned}
$$

up to $\mathcal{O}\left(k^{j \geq 4}\right)$ terms. The coefficients $A_{1}$ and $A_{2}$ are two constants depending only on $k$ through the initial conditions. Although this form is particularly valid as long as $\omega k^{2} \ll z^{\prime \prime} / z$, i.e. when the mode is below its potential, Eq. (25) should formally apply for all times. 
The $k$-dependence of the coefficients $A_{1}$ and $A_{2}$ can be obtained if we notice that, in the time region where Eq. (19) is valid, expansion (25) should coincide with expansion (23). From this relation, we get that $A_{1} \propto k^{3 w / 2}$ and $A_{2} \propto k^{-3 w / 2}$.

As expansion (25) is valid at all times during contraction, the $A_{1}$ and $A_{2}$ dependences hold when the Universe enters the fluid dominated phase just before performing the bounce.

From Ref. 11, the power spectrum is given by

$$
\mathcal{P} \propto k^{3}\left|A_{2}\right|^{2} \propto k^{n_{\mathrm{S}}-1},
$$

yielding the spectral index

$$
n_{\mathrm{S}}=1+3(1-\omega)
$$

in contrast with the result without cosmological constant of Ref. 11,

$$
n_{\mathrm{S}}=1+\frac{12 \omega}{1+3 \omega}
$$

Hence, if we consider the presence of a cosmological constant, an almost scale invariant spectrum is obtained if $w \approx 1$, while in the case without a cosmological constant we have $w \approx 0$.

\section{Discussion}

We have studied the evolution of cosmological perturbations in the context of a bouncing model with a cosmological constant. The main difference is originated from processes much before the bounce, when the cosmological constant becomes important, altering the spectrum of the perturbations before they enter the bounce. This difference comes from the choice of vacuum we have to make in the far past of the contracting phase. Instead of an adiabatic Minkowski vacuum, we chose a Bunch-Davies vacuum in the asymptotic past of the model, where one approaches De Sitter spacetime. We concluded that, in this case, the fluid in the contracting phase must be similar to stiff matter, $w \approx 1$, in order to reproduce an almost scale invariant spectrum of long wavelength perturbations.

\section{Acknowledgments}

We would like to thank Slava Mukhanov and the group of Pequeno Seminário for many useful comments and suggestions. We would also like to thank CNPq of Brazil for financial support.

\section{References}

1. M. Novello and S. E. Perez Bergliaffa, Phys. Rep. 463, 127 (2008).

2. P. Peter and N. Pinto-Neto, Phys. Rev. D 78, 063506 (2008).

3. R. C. Tolman, Phys. Rev. 38, 1758 (1931); G. Murphy, Phys. Rev. D 8, 4231 (1973); M. Novello and J. M. Salim, Phys. Rev. D 20, 377 (1979); V. Melnikov and S. Orlov, Phys. Lett. A 70, 263 (1979). 
4. J. Acacio de Barros, N. Pinto-Neto and M. A. Sagioro-Leal, Phys. Lett. A 241, 229 (1998); R. Colistete Jr., J. C. Fabris and N. Pinto-Neto, Phys. Rev. D 62, 083507 (2000); J. Khoury, B. A. Ovrut, P. J. Steinhardt and N. Turok, Phys. Rev. D 64, $123522(2001)$.

5. V. A. De Lorenci, R. Klippert, M. Novello and J. M. Salim, Phys. Rev. D 65, 063501 (2002); F. G. Alvarenga, J. C. Fabris, N. A. Lemos and G. A. Monerat, Gen. Rel. Grav. 34, 651 (2002); J. C. Fabris, R. G. Furtado, P. Peter and N. Pinto-Neto Phys. Rev. D 67, 124003 (2003); A. Ashtekar, M. Bojowald and J. Lewandowski, Adv. Theor. Math. Phys. 7, 233 (2003).

6. T. Biswas, R. Brandenberger, A. Mazumdar and W. Siegel, JCAP 0712, 011 (2007); L. R. Abramo, P. Peter and I. Yasuda, Phys. Rev. D 81, 023511 (2010); Y.-F. Cai, R. Brandenberger and X. Zhang, JCAP 1103, 003 (2011).

7. R. Brandenberger and F. Finelli, Phys. Rev. D 65, 103522 (2002).

8. L. E. Allen and D. Wands, Phys. Rev. D 70, 063515 (2004).

9. V. Bozza and G. Veneziano, JCAP 09, 007 (2005).

10. F. Finelli, P. Peter and N. Pinto-Neto, Phys. Rev. D 77, 103508 (2008).

11. P. Peter and N. Pinto-Neto, Phys. Rev. D 78, 063506 (2008).

12. C. Lin, R. H. Brandenberger and L. P. Levasseur, JCAP 1104, 019 (2011).

13. P. Peter, E. Pinho and N. Pinto-Neto, J. Cosmol. Astropart. Phys. 07, 014 (2005).

14. P. Peter, E. J. C. Pinho and N. Pinto-Neto, Phys. Rev. D 73104017 (2006).

15. E. J. C. Pinho and N. Pinto-Neto, Phys. Rev. D 76023506 (2007).

16. V. Mukhanov, Physical Foundations of Cosmology (Cambridge University Press, Cambridge, 2005).

17. V. Mukhanov, Introduction to Quantum Effects in Gravity (Cambridge University Press, Cambridge, 2007).

18. V. F. Mukhanov, H. A. Feldman and R. H. Brandenberger, Phys. Rep. 215, 203 (1992). 